Communications in Physics, Vol. 30, No. 4 (2020), pp. 345-353

DOI:10.15625/0868-3166/30/4/14832

\title{
OPTIMIZATION AND CHARACTERIZATION OF PAPER-BASED SERS SUBSTRATES FOR DETECTION OF MELAMINE
}

\author{
NGUYEN THI BICH NGOC ${ }^{1,2, \dagger}$, CHU VIET HA ${ }^{3}$, NGUYEN THI THUY ${ }^{1,2}$, \\ NGUYEN TRONG NGHIA ${ }^{1}$ AND TRAN HONG NHUNG ${ }^{1,2}$
}

${ }^{1}$ Institute of Physics, Vietnam Academy of Science and Technology, 18 Hoang Quoc Viet, Cau Giay, Hanoi, Vietnam

${ }^{2}$ Graduate University of Science and Technology, Vietnam Academy of Science and Technology, 18 Hoang Quoc Viet, Cau Giay, Hanoi, Vietnam

${ }^{3}$ Thai Nguyen University of Education, Tan Thinh Ward, Thai Nguyen city, Thai Nguyen, Vietnam

${ }^{\dagger}$ E-mail: bichngocphysics53@gmail.com

Received 17 February 2020

Accepted for publication 04 August 2020

Published 20 October 2020

\begin{abstract}
A flexible low-cost paper-based surface enhanced Raman scattering (SERS) substrate was successfully manufactured by a direct chemical reduction of silver nanoparticles (AgNPs) onto a common commercially available filter paper. Characterization of fabricated paper-based SERS substrate and the influences of the silver nitrate concentration, type of paper on SERS signal were systematically investigated. In order to fabricate SERS substrates with the highest quality, a suitable one from four different types of filter papers was chosen. The prepared SERS substrates have capability for detecting food toxic chemicals. The test of detecting melamine in aqueous solution was successfully demonstrated with the limit of detection for melamine is $10^{-7} \mathrm{M}$.
\end{abstract}

Keywords: SERS paper, silver nanostructure.

Classification numbers: 82.80.Gk.

\section{INTRODUCTION}

Surface enhanced Raman spectroscopy (SERS) offers a powerful technique for trace chemical and biological detection. The highly sensitive SERS substrate can detect low-abundance molecules by amplification of electromagnetic field through the excitation of localized surface plasmon resonance (LSPR) of plasmonic metal nanostructures. The fabrication of the SERS substrates is one of key processes for SERS analysis. These SERS substrates often are made by using (C)2020 Vietnam Academy of Science and Technology 
a wide variety of materials such as glass, silicon, quartz, alumina, etc. In contrast to other solid SERS substrates, the flexibility and the porous structure of paper allow a boarder range of analytical applications. The molecular detection can be adsorbed on SERS-active substrate by swabbing or dipping on complex sample surfaces. Moreover, the three-dimensional structure of the paper allows both highly specific surface and inter-particle plasmon coupling, further enhancing the SERS signal. On the other hand, the paper has many advantages such as low-cost, eco-friendly and biodegradability [1-3]. So far, paper-based SERS substrates have been fabricated using various approaches, including inkjet printing [4-6], solution dipping [7, 8] and chemical reduction. However, these methods require the complicated process to synthesis of plasmonic nanoparticles on paper, special instruments and multiple reaction steps [9]. Recently, the simple method to prepare the paper-based SERS substrate is directly coated by silver nanoparticles on paper. It often requires a less material consumption, lower energy consumption, simple fabrication instruments and low cost. Thus, several groups in the world have been focused on the strategies for the fabrication of plasmonic nanostructure on paper by direct chemical reduction. For examples, Junhui et al. [10] and Cheng et al. [11] fabricated the paper-based SERS substrates by direct reduction metal ions $(\mathrm{Ag}, \mathrm{Au}, \mathrm{Pd}, \mathrm{Pt})$ which were adsorbed on the paper. Gottesman et al. [12] shown that the in situ synthesis also can be carried out in a single step by sonication of the metal ion solution when immersing filter paper. In these report, the distribution of the nanoparticles on porous paper was optimized by varying the metal ion concentration, sonication time, pretreatment of filter paper, reaction time, temperature [10-13].

In fact, paper-based SERS devices shown that the features of filter paper as porosity, thinness, wicking capability, sample storage capability, concentration of analytes trough lateral-flow paper microfluidics play important roles on the SERS signal intensity [3]. In this work, we present the synthesis of flexibly active SERS substrates by the direct chemical reduction of silver precursor onto a common commercially filter paper in the presence of sodium borohydride $\left(\mathrm{NaBH}_{4}\right)$ as the reducing agent. The effect of silver ions concentrations and different paper types on their morphology of paper-based SERS and their capability to be used for Melamine detection also were reported.

\section{EXPERIMENT}

Silver nitrate $\left(\mathrm{AgNO}_{3}\right)$, sodium borohydride $\left(\mathrm{NaBH}_{4}\right)$, Melamine were purchased from Sigma-Aldrich. We used four different types of filter papers including Whatman filter paper $n_{o} 3$ (GE Healthcare UK), office paper A4 (Thai land), glass fiber filters (GE Healthcare, UK) and a laboratory filter paper $n_{o} 103$ (China). They are denoted by paper P1, paper P2, paper P3 and paper $\mathrm{P} 4$ respectively.

In order to fabricate paper-based SERS substrates, the filter papers were cut into $1.2 \mathrm{~cm} \times$ $1.2 \mathrm{~cm}$ strips and immersed in silver nitrate $\left(\mathrm{AgNO}_{3}\right) 0.1 \mathrm{M}$ in water solvent for $15 \mathrm{~min}$. When the papers were immersed in silver nitrate solution, silver ions were readily impregnated into the cellulose fibers through the pores. Most of the incorporated $\mathrm{Ag}^{+}$ions were bound to cellulose macromolecules probably via electrostatic (i.e ion-dipole) interactions [10] Then, the filter papers were dipped into $150 \mu \mathrm{l}$ sodium borohydride $\left(\mathrm{NaBH}_{4}\right) 0.2 \mathrm{M}$ solution to form silver nanostructures for $5 \mathrm{~min}$. Finally, the papers change into dark green and were cleaned with deionized water and dried at room temperature. Ag ions were reduced to $\mathrm{Ag}$ atoms and transformed into spherical silver nanoparticles by $\mathrm{NaBH}_{4}$ like the growth nanoparticles on solution. The growth mechanism 
of reduction process in solution by a strong reductant like $\mathrm{NaBH}_{4}$ is governed by coalescence closely through four stages was given by Polte et al. [14]. However, when reduced on paper fibers, these silver nanoparticles continue to grow by the mechanism of non-classical particles mediated growth [15].

A series of melamine solutions with different concentrations were prepared by dissolving the melamine powders in water. A paper-based SERS substrate $(3 \mathrm{~mm} \times 3 \mathrm{~mm})$ was dropped $10 \mu 1$ melamine solution and dried in air at room temperature before SERS analysis. Raman spectra were recorded by a Raman spectrometer at our laboratory using He-Ni laser with the wavelength of $633 \mathrm{~nm}$, laser power of $10 \mathrm{~mW}$ with integration time of $3 \mathrm{~s}$ and laser spot size of $14 \mu \mathrm{m}$.

\section{RESULTS AND DISCUSSION}

\section{Characterization of the paper-based SERS substrates}
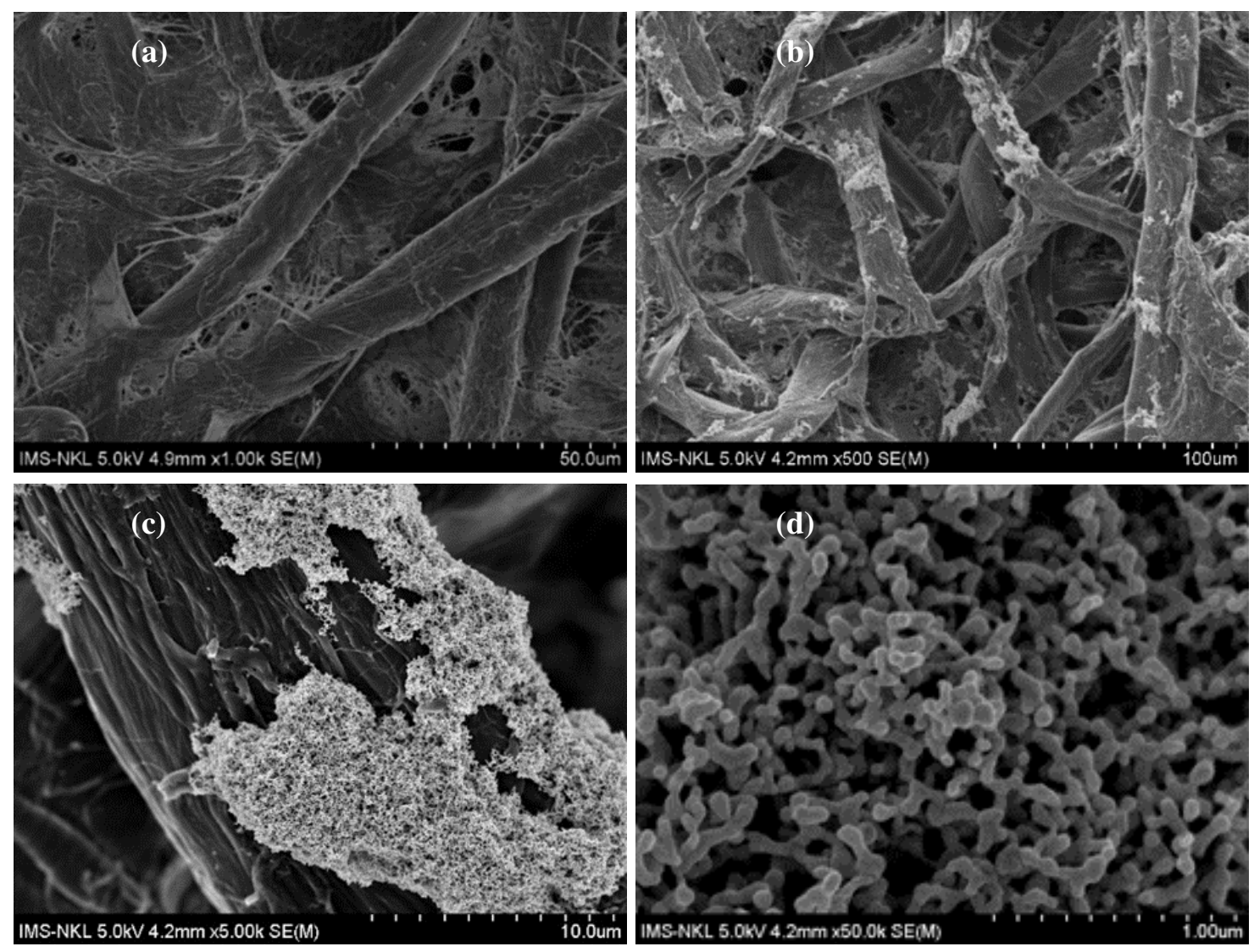

Fig. 1. The FE-SEM image of paper P1 (a), Ag- filter paper (b) and the clusters of silver nanoparticles on filter paper surface (c), (d).

Both UV-VIS and SEM analyses of the substrates were conducted. The SEM images in Fig. 1 exhibit that $\mathrm{Ag}$ structures with numerous pieces of $\mathrm{Ag}$ were formed on the filter paper. 
The average piece size is $40-50 \mathrm{~nm}$. And these pieces joined together to form discrete corals on filter paper fibers. Although the distribution of silver clusters on the filter paper is not uniform (Fig. 1b), it also does not influence on the SERS signal results. Because of the large number of silver clusters in the focused region of the laser trace diameter, it allows averaging over a multitude of silver aggregates, lowering variability and enabling quantitative results.

This formation of silver coral structures on paper fibers can be explained by the Orientated Attachment $(\mathrm{OA})$ mechanism. In order to reduce the overall energy to minimum during the coalescence of silver pieces, they attach through the facets with the highest surface energy or surface area to decrease the overall surface energy. Besides the strong reducing agent $\mathrm{NaBH}_{4}$, the fabrication speed of small silver nanoparticles is very fast, about a few hundred seconds. These small silver pieces are fabricated on the surface of filter paper with the distance between them is very small. Under the small distance between the nanocrystals, the van der Waals interaction enhanced. The bonding force takes full effect and rapidly drivers the nanocrystals to rotate and coalesce [15].

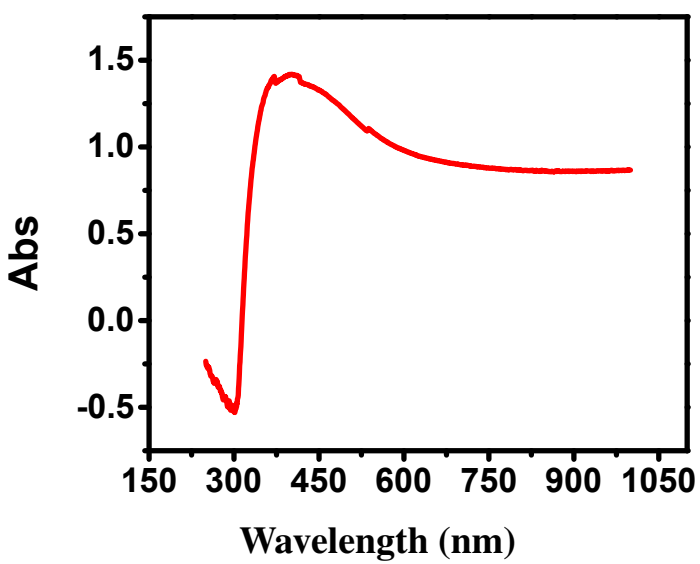

Fig. 2. The scattering spectra of Ag-filter paper.

Therefore silver ions can be adsorbed initially on uniform paper fibers but after being reduced to small silver particles, there are not electrostatic binding forces between these silver particles and paper fibers. At this time, the Van der Waals attraction of these pieces is stronger, so to get the lowest surface energy, they are joined together to form discrete corals on filter paper fibers.

The UV-Vis extinction spectrum of a paper-based SERS substrate was shown in Fig. 2. A clear absorption peak occurred at $420 \mathrm{~nm}$. Besides, the UV-Vis spectra peaks corresponding to high-order multipole plasmon resonance modes were observed and lead the expanding strong absorption band at a long wavelength.

\section{Optimization of the paper-based SERS substrates}

The paper-based SERS substrates were prepared in different concentrations of silver ions solution. The paper P1 was used to fabricate SERS substrates with silver nitrate $\left(\mathrm{AgNO}_{3}\right)$ concentration from $0.05 \mathrm{M}$ to $0.35 \mathrm{M}$. After that, $10 \mu \mathrm{l}$ of melamine solution of $10^{-4} \mathrm{M}$ was deposited on these substrates. The SERS spectra were plotted in Fig. 3(a) and the dependence of SERS signal intensity on the concentration of $\mathrm{AgNO}_{3}$ was shown in Fig. 3(B). The predominant band in the Raman spectra of melamine located at $672 \mathrm{~cm}^{-1}$ can be assigned to the in-plane ring breathing II mode, which involves an in-plane deformation of the triazine ring with the vibration of the amino nitrogen atoms. The SERS spectrum of melamine in Fig. 3a showed that the vibrations at $672 \mathrm{~cm}^{-1}$ are shifted to $700 \mathrm{~cm}^{-1}$. This change of peak position indicated that a strong interaction existed between triazine ring and silver structures on fiber paper [16].

Fig. $3 \mathrm{~b}$ also showed the variation of SERS signal intensity at $700 \mathrm{~cm}^{-1}$ with different concentration of $\mathrm{AgNO}_{3}$. This is due to the dependence of SERS signal on the distribution and shape 
of silver nanostructures which were formed on the filter paper. The graph of characteristic peak intensity of Melamine vs. concentration of $\mathrm{AgNO}_{3}$ was shown in Fig.3b. With low silver ion concentration $(0.05 \mathrm{M}, 0.1 \mathrm{M})$, the silver nanoparticles on paper were obtained with small and sparse aggregation. The density of silver structures in paper substrates increased with the increasing the silver concentration. Therefore, the SERS intensity also strongly increases with increasing concentration of $\mathrm{AgNO}_{3}$. After the concentration of $\mathrm{AgNO}_{3}$ increase to 0.25, the number of gaps between silver nanoparticles decreases and the SERS signal of melamine is lower with higher concentration of $\mathrm{AgNO}_{3}$.
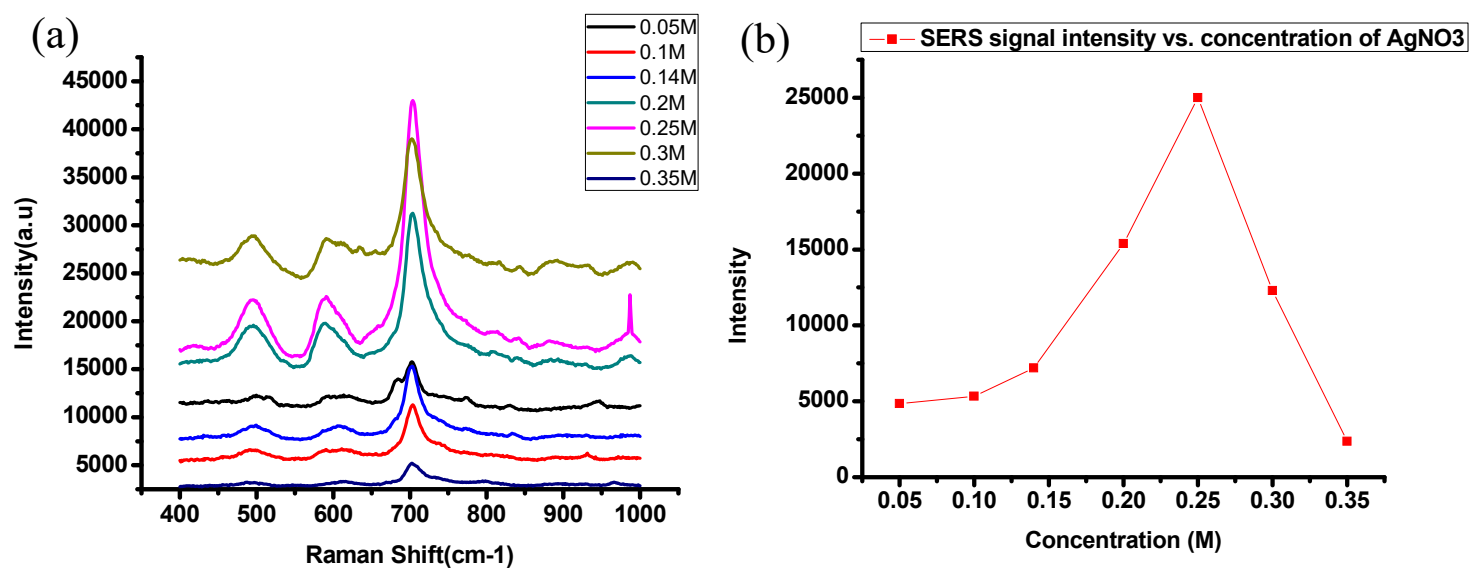

Fig. 3. Measured Raman spectra for Melamine $\left(10^{-4} \mathrm{M}\right)$ with different concentrations of silver nitrate (a) and the graph of SERS signal intensity vs. concentration of $\mathrm{AgNO}_{3}$ (b).

Four types of paper were used to demonstrate the influences of their structure on SERS signal. SEM images of prepared SERS substrates were showed in Fig. 5. The silver structures are formed from the aggregation of small silver nanoparticles. Three types of filter paper P1, P2, and P4 are made from cellulose fiber while the paper P3 is glass fibers The SEM images showed that the silver clusters can be observed for all papers; however, office paper provided to outnumber of silver nanostructures on paper fiber. In case of using glass fiber filter the formation of silver structures did not take place on the fibers but they formed large silver clusters in porosities of filter. In contrast, these silver clusters are formed on the fiber for other 3 types of paper. It can be explained the crystals of the cellulose are built up through the hydrogen bonding between $\mathrm{OH}$ functional group. The formation of silver structures on filter paper depends on the bonding between silver ions and cellulose fibers. The glass fiber filter have a low absorption ability of the metal ions because glass fibers do not contain functional group on the surface The silver clusters were not formed on the fibers as with other cellulose papers.

The coupling of small pieces of silver to form silver clusters on the four types of paper is also different. The SERS substrate using paper P1 has more gaps between silver nanostructures than other types of paper. The narrow gaps between these silver nanostructures have a high surface plasmon resonance field. Therefore, the SERS signal of melamine $10^{-4} \mathrm{M}$ on the SERS substrate using paper P1 is higher (Fig. 4, Table 1). The SERS intensity of melamine on using paper P3 and 
P4 substrates are also similar with using paper P1. Although the silver clusters on office paper are more evenly than other three types of paper, the porosity of silver nanostructures is low, resulting in not many hot spots. The SERS spectrum of Melamine on this substrate is not as high as other structures but the SERS spectrum of Melamine collected at 10 randomly chosen spots on SERS substrate based on office paper is the best uniform. The larger amount of silver clusters on office paper surface is similar to other reports of paper P1 [17]. In addition, the SERS spectra of nonmelamine paper types show that the spectrum of paper P1 has fewer peaks of impurities than the paper P2. These peaks will interfere with the detection of substances in low concentrations on these substrates.

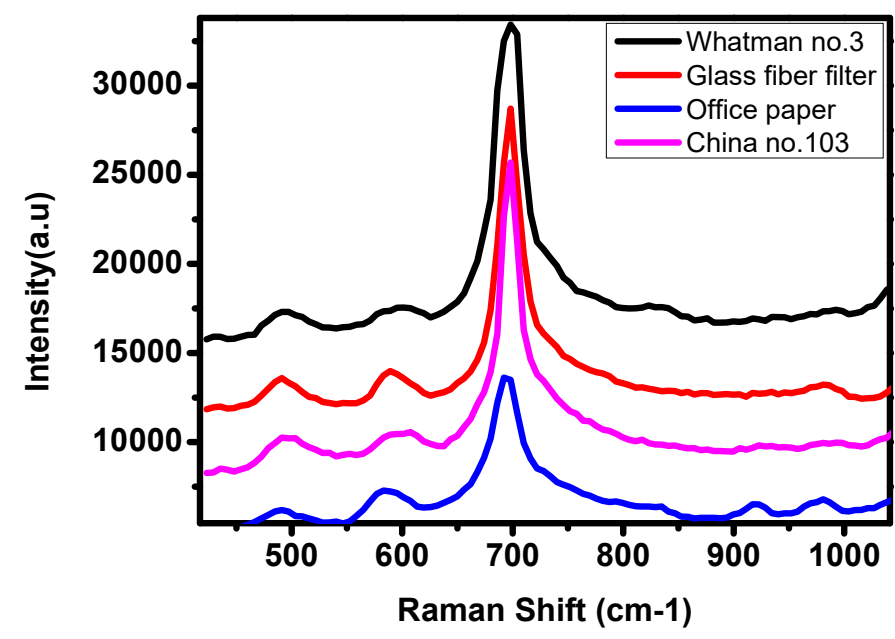

Fig. 4. Raman spectra of Melamine solution $\left(10^{-4} \mathrm{M}\right)$ with different types of filter paper.

Table 1. Data SERS peak intensity of Melamine in concentration of $10^{-4} \mathrm{M}$ with different kinds of filter paper.

\begin{tabular}{|c|c|c|}
\hline Paper & Type of Paper & $\begin{array}{c}\text { Peak intensity at } \mathbf{7 0 0} \mathbf{c m}^{-1} \\
\text { of Melamine in concentration of } \mathbf{~ 1 0}^{-4} \mathbf{M}\end{array}$ \\
\hline P1 & $\begin{array}{c}\text { Whatman filter paper no 3 } \\
\text { GE Healthcare companies, UK }\end{array}$ & 16702 \\
\hline P2 & $\begin{array}{c}\text { Office paper, A4 paper, } \\
\text { Double A, Thailand }\end{array}$ & 7623 \\
\hline P3 & $\begin{array}{c}\text { Glass fiber filter GE } \\
\text { Healthcare companies, UK }\end{array}$ & 16284 \\
\hline P4 & Filter paper no103, China & 16209 \\
\hline
\end{tabular}



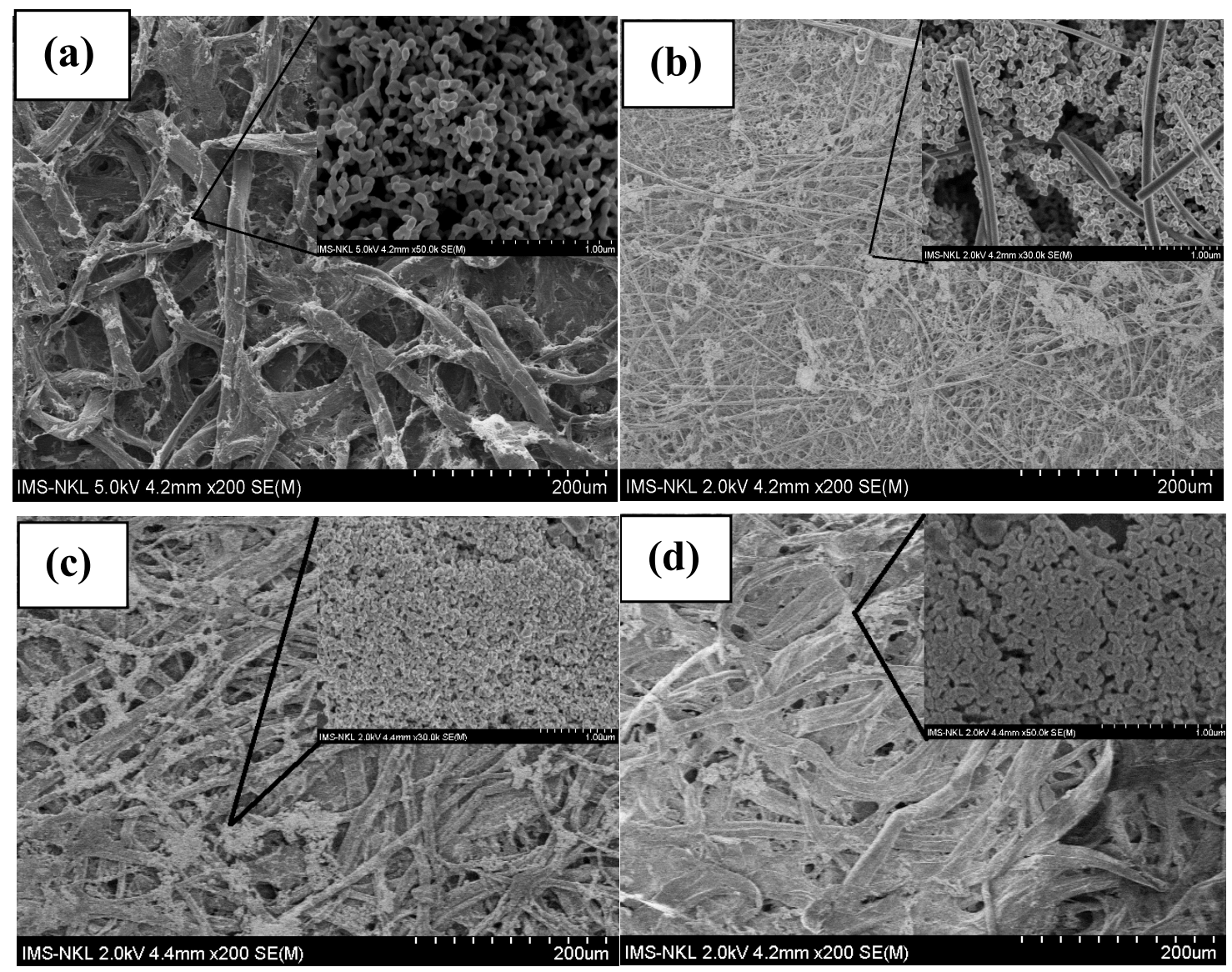

Fig. 5. The FE-SEM image of the SERS substrates based on the paper P1(a), the paper P3 (b), the paper P2(c) and the paper P4 (d).

\section{The limits of detection for melamine}

The fabricated SERS substrate was used for the detection of melamine in solution at low concentration to find the detection limit. Raman spectra were measured for melamine solution at different concentrations from $10^{-4} \mathrm{M}$ to $10^{-7} \mathrm{M}$. As shown in Fig. 6 the in-plane deformation vibration of triazine ring at $700 \mathrm{~cm}^{-1}$ of melamine can be observed in SERS spectra with concentration of $10^{-7} \mathrm{M}$. It can be seen from Fig. 5 that melamine at concentration $10^{-7} \mathrm{M}$ can be detected by this technique. The average SERS enhancement factor (EF) was calculated from the equation:

$$
E F=\left(\frac{I_{S \mathrm{ER} S}}{I_{\text {Raman }}}\right)\left(\frac{N_{\text {Raman }}}{N_{S E R S}}\right)
$$

where $I_{S E R S}$ is the SERS intensity of a particular Raman line of Melamine and $I_{\text {Raman }}$ is Raman intensity of the Melamine powder on paper. $N_{S E R S}$ corresponds to the estimated number of molecules 
contributing to the SERS signal, while $N_{\text {Raman }}$ is the number of molecules contributing to the Raman signal from the non-SERS surface. They are determined by the relation:

$$
\begin{aligned}
N_{S E R S} & =\eta N_{A} V C_{S E R S} \frac{V_{\text {laser }}}{V_{\text {SERS }}} ; \\
N_{\text {Raman }} & =N_{A} \frac{d_{\text {Melamine }}}{M} V_{\text {laser }} ; \\
\frac{N_{\text {Raman }}}{N_{\text {SERS }}} & =\frac{d_{\text {Melamine }} V_{\text {SERS }}}{\eta M V C_{S E R S}},
\end{aligned}
$$

where $N_{A}$ is the Avogadro number, $V$ is total volume of solution spread on the substrate $(10 \mu \mathrm{L})$, $\mathrm{V}_{\text {laser }}$ is the volume of the laser, $V_{S E R S}$ is the total volume of the SERS substrate covered by the drop of Melamine solution $(3 \mathrm{~mm} \times 3 \mathrm{~mm} \times 0.39 \mathrm{~mm})$.
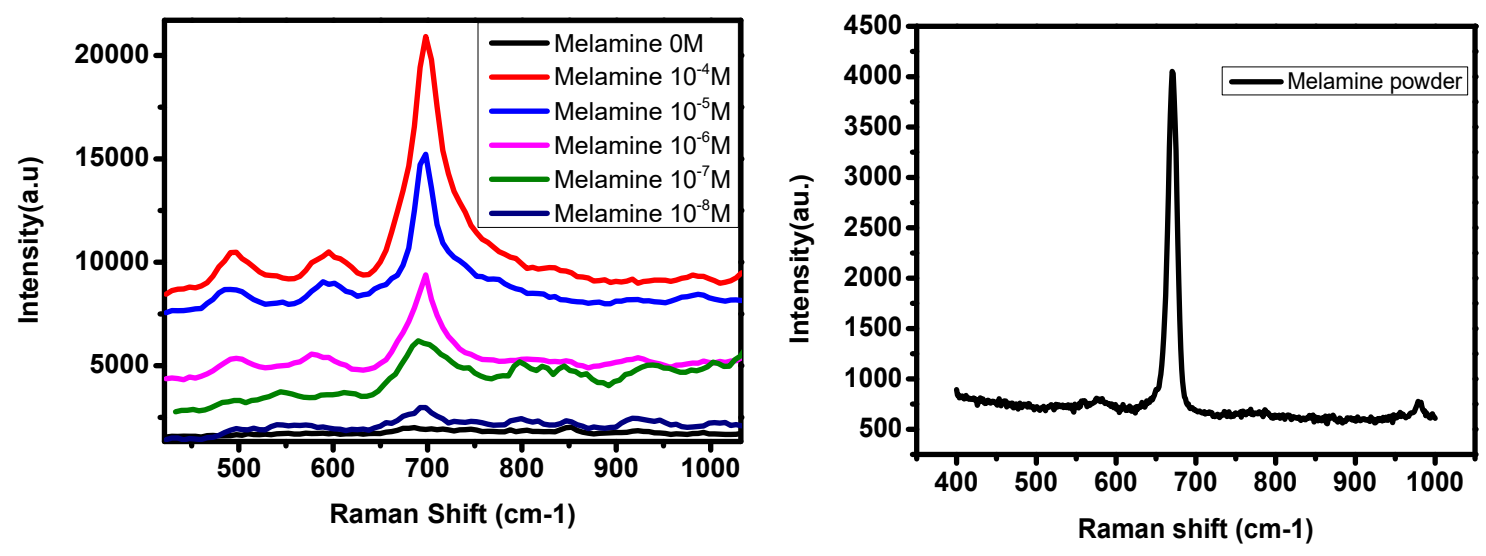

Fig. 6. Limit of detection for melamine (left) and Raman spectral of the Melamine power measured on glass (right).

It can be observed from the SEM images that the total active volume with SERS only accounts of $20 \%$ in the total volume of laser spot $(\eta=0.2)$. Melamine powder was put on nonSERS substrate and considered as uniform covering in the total volume of laser spot. By applying equations and the value in Table 2 , the enhancement factor of $2.2 \times 10^{8}$ was found. This work may provide a potential technique for detection of low concentration analysis.

Table 2. Data the value to caculate the average SERS enhancement factor (EF).

\begin{tabular}{|c|c|c|c|c|c|c|}
\hline $\begin{array}{c}I_{\text {SERS }}(\mathrm{au}) \\
\text { Melamine } 10^{-7} \mathrm{M}\end{array}$ & $\begin{array}{c}I_{\text {Raman }}(\mathrm{au}) \\
\text { Melamine powder }\end{array}$ & $\begin{array}{c}V_{\text {SERS }} \\
\left(\mathrm{m}^{2}\right)\end{array}$ & $\begin{array}{c}d_{\text {Melamine }} \\
\left(\mathrm{kg} / \mathrm{m}^{3}\right)\end{array}$ & $\begin{array}{c}M_{\text {Melamine }} \\
(\mathrm{g} / \mathrm{mol})\end{array}$ & $\begin{array}{c}\mathrm{V} \\
(\mu \mathrm{l})\end{array}$ & $\begin{array}{c}C_{\text {SERS }} \\
(\mathrm{M})\end{array}$ \\
\hline 2987 & 3459 & $3.51 \times 10^{-9}$ & 1574 & 126.12 & 10 & $10^{-7}$ \\
\hline
\end{tabular}




\section{CONCLUSION}

We successfully prepared paper-based SERS substrates by directly fabricated the silver nanostructures on filter paper. The silver nanoparticle size is about 40-50 nm and aggregated together to form discrete corals on paper. In four type papers the Whatman filter paper grade 3 with concentration of $\mathrm{AgNO}_{3}$ solution $0.25 \mathrm{M}$ is optimal for SERS signal. The limit of detection of our substrates was $10^{-7} \mathrm{M}$ for Melamine.

\section{ACKOWLEDGMENTS}

This work was supported by Vietnam Academy of Science and Technology (VAST) under contract number 1412/HĐ-VHL (2016-2019).

\section{REFERENCES}

[1] Wei Wu, Li Liu, Zhigao Dai, Juhua Liu, Shuanglei Yang, Li Zhou, Xiangheng Xiao, Changzhong Jiang and Vellaisamy A. L. Roy, Sci. Rep. 5 (2014) 10208.

[2] Wenxian Wei and Quingli Huang, Spetrochimica Acta Part A 179 (2017) 211.

[3] Maria João Oliveira, Pedro Quaresma, Miguel Peixoto de Almeida, Andreia Araújo, Eulália Pereira, Elvira Fortunato, Rodrigo Martins, Ricardo Franco and Hugo Águas, Sci. Rep. 7 (2017) 2480

[4] Wei W. Yu and Ian M. White, Anal Chem. 82 (2010) 9626.

[5] A. Eshkeiti, B.B. Narakathu, A.S.G. Reddy, A. Moorthi and M.Z. Atashbar, Procedia Engineering 25 (2011) 338

[6] Wei Zhang, Bowei Li, Lingxin Chen, Yunqing Wang, Dingxue Gao, Xuehua Ma and Aiguo Wu, Anal. Methods 6 (2014) 2066

[7] R. Tantra, R.J. Brown, M.J. Milton and D.Gohil, Appl. Spectrosc. 9 (2008) 992.

[8] M. K. Fan, G. F. S. Andrade and A. G. Brolo, Anal. Chim. Acta 7 (2011) 693.

[9] Vo Thi Nhat Linh, Jungil Moon, Chae Won Mun, Vasanthan Devaraj, Jin-Woo Oh, Sung-Gyu Park, Dong-Ho Kim, Jaebum Choo, Yong-Ill Lee, Ho Sang Junga, Sens. Actuators: B 291 (2019) 369.

[10] Junhui He, Toyoki Kunitake and Aiko Nakao, Chem. Mater. 15 (2003) 4401.

[11] Min-Liang Cheng, Bo-Chan Tsai and Jyisy Yang, Anal. Chim. Acta 708 (2011) 89.

[12] Ronen Gottesman, Sourabh Shukla, Nina Perkas, Leonid A. Solovyov, Yeshayahu Nitzan, and Aharon Gedanken, Langmuir 27 (2011) 720.

[13] Silvia Dalla Marta, Chiara Novara, Fabrizio Giorgis, Alois Bonifacio, and Valter Sergo, Materials 10 (2017) 1365.

[14] Polte, JACS Nano 6 (2012) 5791

[15] Hongjun You and Jixiang Fang, Nano Today 11 (2016) 145.

[16] Panneerselvan Rajapandiyan, Wei-Li Tang and Jyisy Yang, Food Control 56 (2015) 155

[17] Maria João Oliveira, Pedro Quaresma, Miguel Peixoto de Almeida, Andreia Araújo, Eulália Pereira, Elvira Fortunato, Rodrigo Martins, Ricardo Franco and Hugo Águas, Sci. Rep. 7 (2017) 2480. 